\title{
Nitrogen dioxide induces apoptosis and proliferation but not emphysema in rat lungs
}

\author{
Heinz Fehrenbach, Gregor Zimmermann, Ellen Starke, Vlad A Bratu, Dominik Conrad, Ali Ö Yildirim, \\ Antonia Fehrenbach
}

See end of article for authors' affiliations

Correspondence to

Professor H Fehrenbach

Clinical Research Group

Chronic Airway Diseases,

Department of Internal

Medicine (Respiratory

Medicine), Philipps

University, Baldingerstrasse,

D-35043 Marburg,

Germany; heinz.

fehrenbach@staff.uni-

marburg.de

Received 14 March 2006

Accepted 6 November 2006

\begin{abstract}
Background: Apoptosis of alveolar septal cells has been linked to emphysema formation. Nitrogen dioxide, a component of cigarette smoke, has been shown to induce alveolar epithelial cell apoptosis in vitro. It is hypothesised that exposure of rats to nitrogen dioxide may result in increased alveolar septal cell apoptosis in vivo with ensuing emphysema-that is, airspace enlargement and loss of alveolar walls.

Methods: Fischer 344 rats were exposed to 10 ppm nitrogen dioxide for $3,7,21$ days or 21 days followed by 28 days at room air. Age-matched control rats were exposed to room air for 3, 21 or 49 days. Lungs fixed at $20 \mathrm{~cm}$ fluid column, embedded in paraffin wax, glycol methacrylate and araldite, were analysed by design-based stereology. Alveolar septal cell apoptosis (transferase dUTP nick end labelling assay, active caspase 3) and proliferation (Ki-67), airspace enlargement, total alveolar surface area, and absolute alveolar septal volume as well as the ultrastructural composition of the alveolar wall were quantified.

Results: Nitrogen dioxide resulted in an eightfold increase in alveolar septal cell apoptosis at day 3 and a 14fold increase in proliferation compared with age-matched controls. Airspace enlargement, indicated by a $20 \%$ increase in mean airspace chord length, was evident by day 7 but was not associated with loss of alveolar walls. By contrast, nitrogen dioxide resulted in an increase in the total surface area and absolute volume of alveolar walls comprising all compartments. The ratio of collagen to elastin, however, was reduced at day 21. Lungs exposed to nitrogen dioxide for 21 days exhibited quantitative structural characteristics as seen in control lungs on day 49.

Conclusions: Nitrogen dioxide exposure of rats results in increased alveolar septal cell turnover leading to accelerated lung growth, which is associated with an imbalance in the relative composition of the extracellular matrix, but fails to induce emphysema.
\end{abstract}

$\mathrm{E}$ mphysema is defined as the "abnormal permanent enlargement of the airspaces distal to the terminal bronchioles, accompanied by destruction of their walls". ${ }^{1}$ The pathogenetic pathways leading to emphysema are still a matter of debate. At present there are two major concepts which, although not necessarily contradictory, have clearly different perspectives of the sequence of pathogenetic events. The classical concept focuses on the inflammation-associated imbalance of proteases and antiproteases. This is thought to be the primary cause of degradation of matrix components, which subsequently results in the loss of alveolar septal walls. ${ }^{2}$ On the basis of recent studies, an alternative concept has been developed, which focuses on the apoptosis of endothelial and/ or alveolar epithelial cells as being the primary event in the pathogenesis of emphysema. ${ }^{3}$

Experimental induction of the apoptosis of pulmonary endothelial cells-for example, by blockade of vascular endothelial growth factor receptor $2^{5}$ or lung-targeted inactivation of vascular endothelial growth factor, ${ }^{6}$ or of alveolar epithelial cells by transfer of active caspase $3^{7}$ or cigarette smoke exposure ${ }^{8}$-were shown to result in airspace enlargement. Additional evidence for the importance of apoptosis in the pathogenesis of emphysema comes from several human studies, which showed increased levels of apoptotic alveolar septal cells in patients with emphysema. ${ }^{9-11}$ Notably, programmed cell death appeared to be predominant in alveolar epithelial cells of patients with emphysema, whereas apoptosis of endothelial cells was observed less frequently. ${ }^{10}{ }^{11}$

The major risk factors for chronic obstructive pulmonary disease (COPD), which comprises chronic bronchitis and emphysema, are cigarette smoking and indoor air pollution from burning fuels. ${ }^{12}$ Until now, however, studies of experimental animal models based on induction of emphysema by inhalation of, for example, cigarette smoke have shown contradictory results regarding the involvement of apoptosis of alveolar septal cells in the development of emphysema. ${ }^{8}{ }^{13}$

Nitrogen dioxide is an important component of cigarette smoke with reported emissions of up to $0.73 \mathrm{mg}$ nitrogen dioxide per cigarette. ${ }^{14}$ Exposure of rats to nitrogen dioxide results in airway inflammation which, as in human COPD, is dominated by alveolar macrophages and neutrophilic granulocytes. $^{15} 16$ Further, nitrogen dioxide induces apoptosis of alveolar epithelial cells in vitro with actively dividing cells and cells at the leading edge of a wound being particularly susceptible. ${ }^{17}$ As short-term exposure of rats to nitrogen dioxide in vivo results in an initial phase of alveolar epithelial injury followed by a phase of epithelial repair, ${ }^{18}$ we hypothesised that the exposure of rats to a nitrogen dioxide-containing atmosphere may result in an increase in apoptosis of alveolar epithelial cells in vivo with ensuing emphysema.

\section{MATERIALS AND METHODS \\ Experimental protocol}

Forty-two male Fischer 344 rats (Charles River, Sulzfeld, Germany), 181 (5) g body weight, 8-10 weeks of age, were divided into seven groups. Three groups $(\mathrm{n}=6$ each) were exposed for 3, 7 or 21 days ( $23 \mathrm{~h}$ /day, 7 days/week) to an atmosphere containing $10 \mathrm{ppm}$ nitrogen dioxide (Messer Griesheim, Duisburg, Germany) as described elsewhere. ${ }^{19}$ An

Abbreviations: COPD, chronic obstructive pulmonary disease; TUNEL, transferase dUTP nick end labelling 
Table 1 Body weights and food consumption

\begin{tabular}{|c|c|c|c|c|}
\hline Experimental group & $\begin{array}{l}\text { Exposure time } \\
\text { (days) }\end{array}$ & $\begin{array}{l}\text { Number of } \\
\text { animals }\end{array}$ & Body weight (g) & $\begin{array}{l}\text { Mean food consumption } \\
\text { (g/day) }\end{array}$ \\
\hline 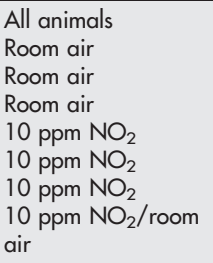 & $\begin{array}{l}0 \\
3 \\
21 \\
49 \\
3 \\
7 \\
21 \\
21 \\
+28\end{array}$ & $\begin{array}{l}42 \\
4 \\
8 \\
6 \\
6 \\
6 \\
6 \\
6\end{array}$ & 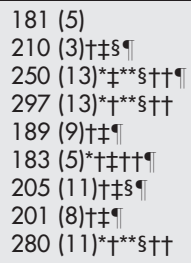 & 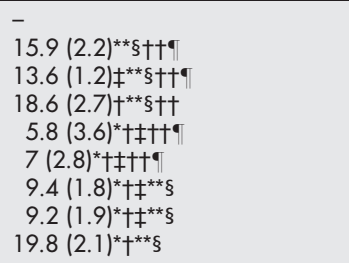 \\
\hline \multicolumn{5}{|c|}{$\begin{array}{l}\text { Values are expressed as mean (SD). } \\
\text { Significant differences among groups at } \mathrm{p}<0.05 \text { (one way analysis of variance followed by post hoc pairwise multiple } \\
\text { comparisons procedures, Tukey's test) are indicated as follows: } \\
\text { *vs room air } 3 \text { days. } \\
\text { †vs room air } 21 \text { days. } \\
\text { †vs room air } 49 \text { days. } \\
\S v \mathrm{NO}_{2} 7 \text { days. } \\
\text { Ivs } \mathrm{NO}_{2} / \text { room air. } \\
\text { **vs } \mathrm{NO}_{2} 3 \text { days. } \\
\text { ††vs } \mathrm{NO}_{2} 21 \text { days. }\end{array}$} \\
\hline
\end{tabular}

additional group $(n=6)$ was exposed to nitrogen dioxide for 21 days followed by exposure to room air for 28 days. The concentration of nitrogen dioxide was monitored by a nitrogen dioxide-sensitive electrochemical element (ECS 102-1, MP Sensor System, Munich, Germany). Age-matched control groups $(n=4-8)$ were exposed to room air for 3, 21 or 49 days. Mean food consumption per day was monitored. Body weights were measured at the end of each exposure period. The animal experiments were approved by the regional government (Regierungspräsidium Giessen, Dezernat V.54, Giessen, Germany).

\section{Fixation and tissue sampling}

Immediately after exposure, rats were killed. Lungs were fixed by airway instillation with $4 \%$ phosphate-buffered paraformaldehyde at a pressure of $20 \mathrm{~cm}$ fluid column. After overnight immersion into fresh fixative, lung volume was determined by fluid displacement, and two to three fractions of lung slices were collected by systematic uniform random sampling as described previously. ${ }^{20}$

\section{Embedding in glycol methacrylate and araldite}

One fraction of lung slices and a systematic uniform random subsample of tissue blocks from the second fraction of slices were post-fixed in $1 \%$ glutardialdehyde, $1 \%$ paraformaldehyde in $0.1 \mathrm{M}$ sodium cacodylate buffer followed by osmication and en bloc staining with aqueous uranyl acetate before dehydration. Complete lung slices were embedded in glycol methacrylate for stereological analysis of emphysema. Tissue blocks subsampled from the second fraction of slices of lungs exposed for 3-21 days to nitrogen dioxide or room air were embedded in araldite for quantification of the components of the alveolar wall by means of transmission electron microscopy.

\section{Embedding in paraffin wax}

The third fraction of lung slices was dehydrated and embedded in paraffin wax for stereological analysis of cell proliferation and apoptosis, and identification of apoptotic cell types by means of double labelling.

\section{Demonstration of cell proliferation and apoptosis}

Cell proliferation was assessed by immunohistochemistry using the proliferation marker Ki-67 as described elsewhere. ${ }^{21}$ Apoptosis was assayed using terminal transferase dUTP nick end labelling (TUNEL) and immunohistochemistry for active caspase $3 .^{22}$ To identify the phenotype of apoptotic cells, double staining by TUNEL assay and immunohistochemistry for epithelial and endothelial cell markers were performed as described previously. ${ }^{22}$

\section{Stereology at the light microscopical level}

Richardson-stained glycol methacrylate sections were used for stereological quantification of airspace enlargement (independent measurements of mean airspace chord length, alveolar surface density and volume-weighted mean airspace volume), total alveolar surface area and total volume of alveolar septal wall tissue according to standard techniques by point and intersection counting. ${ }^{20}$ In addition, point counting was performed on glycol methacrylate sections to quantify the total volumes of inflammatory cells, alveolar macrophages and intraalveolar polymorphonuclear granulocytes. Paraffin wax sections stained for Ki-67 and for DNA single strand breaks by TUNEL assay were used for stereological quantification of cell proliferation and apoptosis, respectively. ${ }^{21}{ }^{22}$ Briefly, the fractions of alveolar wall occupied by proliferating or apoptotic alveolar septal wall cells were assessed by counting the number of test line intersections with alveolar wall associated with cells stained for Ki-67 or TUNEL, respectively, relative to the total number of intersections with alveolar wall surface. As we were mainly interested in the effect of exposure on the alveolar septal wall architecture, only those cells were included in the analysis that were part of the alveolar septal wall, whereas free apoptotic or proliferating cells present in the alveolar air space were not considered. Analyses were performed on a computerbased Olympus BX 51 light microscope equipped with a CastGrid 2.01 system (Olympus, Denmark).

\section{Stereology at the electron microscopical level}

Ultra thin sections of araldite-embedded tissue blocks were collected on $200 \mu \mathrm{m}$ mesh grids and stained accordingly. ${ }^{23}$ For quantification of the volume fractions of the components of the alveolar wall-that is, alveolar epithelium, capillary endothelium, and interstitial tissue including elastin and collagen deposition-point counting was performed on each upper left corner of the grid. A transparent sheet with 140 equidistant test points was superimposed onto a television monitor, which was connected to a Zeiss EM 900 transmission electron microscope (Carl Zeiss, Oberkochen, Germany), and used for counting all points hitting the respective component relative to the total number of hits on the alveolar wall at a final magnification of 

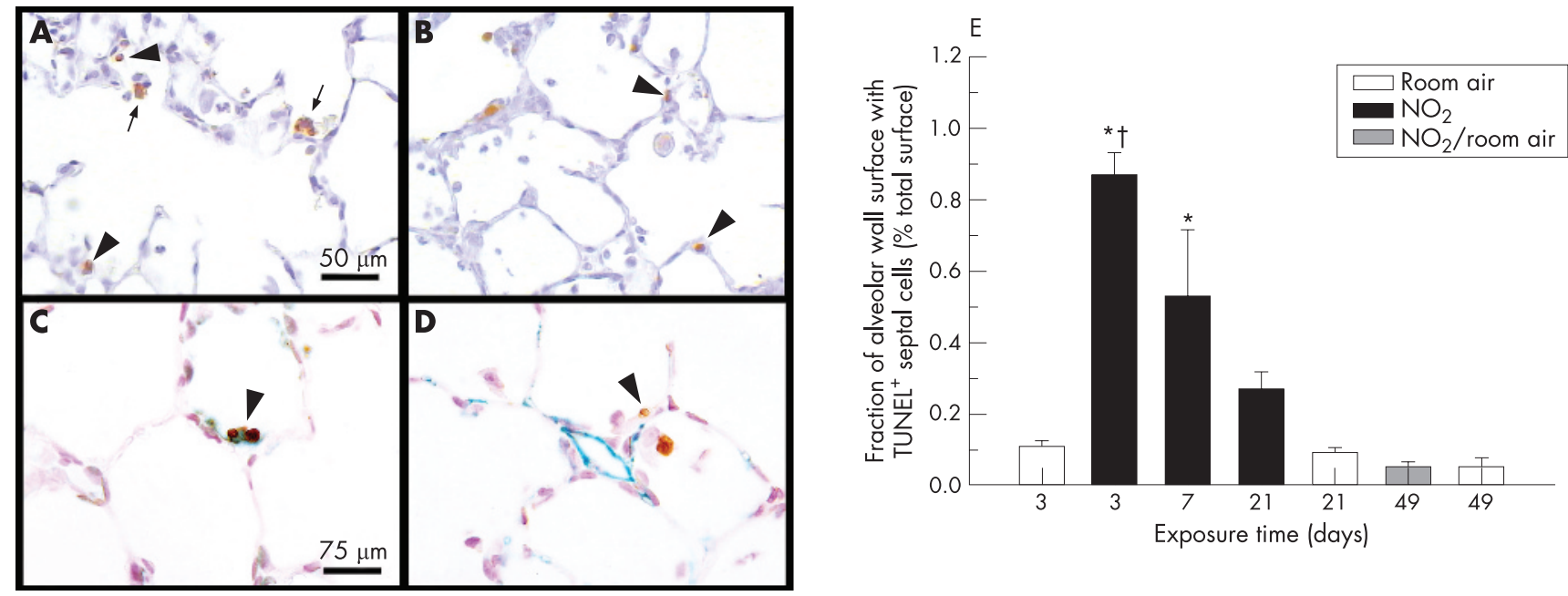

Figure 1 Apoptosis of alveolar septal cells. Apoptotic alveolar septal wall cells (arrowheads) are shown by (A) TUNEL assay and (B) indirect immunohistochemistry against active caspase 3 in lungs exposed to nitrogen dioxide for 3 days; arrows indicate alveolar macrophages with engulfed apoptotic bodies. (C) Apoptotic cells are identified as alveolar epithelial type II cells by double staining using TUNEL assay (brown staining) and indirect immunohistochemistry against surfactant protein D (greenish staining), whereas (D) co-staining against aquaporin 1 (greenish staining) shows that endothelial cells do not undergo apoptosis (brown staining); lungs were exposed to nitrogen dioxide for 3 days. (E) Quantification of TUNEL assay (as shown in A) shows a considerable increase in the apoptosis of structure forming alveolar septal wall cells at day 3 and day 7 of exposure to nitrogen dioxide. The magnifications used in (A) and (B), and in (C) and (D), were the same. Significant differences between groups ( $<<0.05$, one way analysis of variance followed by post hoc multiple comparisons according to Tukey's test) are indicated as follows: "vs all groups exposed to room air and vs $\mathrm{NO}_{2} /$ room air; †vs $\mathrm{NO}_{2} 21$ days.

60.632. As described earlier, ${ }^{24}$ the total volume of each component was obtained by multiplying the volume fraction by the volume of the reference space, the alveolar wall.

\section{Statistics}

Mean (SD) values are given unless indicated otherwise. Differences between experimental groups were tested for significance with parametric one-way analysis of variance followed by post hoc multiple comparisons (Tukey's test) provided that normality and equal variance were given at $\mathrm{p}>0.1$. Otherwise, nonparametric one-way analysis of variance on ranks was used. Student's $t$ test was used to test for differences between pairs of groups. Significance of differences between groups was considered for $\mathrm{p}<0.05$. Spearman rank order correlation analysis was performed to test for relationships between proliferation, apoptosis and inflammation. All statistical analyses were performed using the SigmaStat V.3.1 software program. $p$ Values $<0.05$ were considered to be significant.

\section{RESULTS}

\section{General effects of nitrogen dioxide exposure}

The initial mean body weight of the animals before exposure was 181 (5) g. Rats exposed to nitrogen dioxide showed no significant gain in body weight during the first week of exposure (table 1).
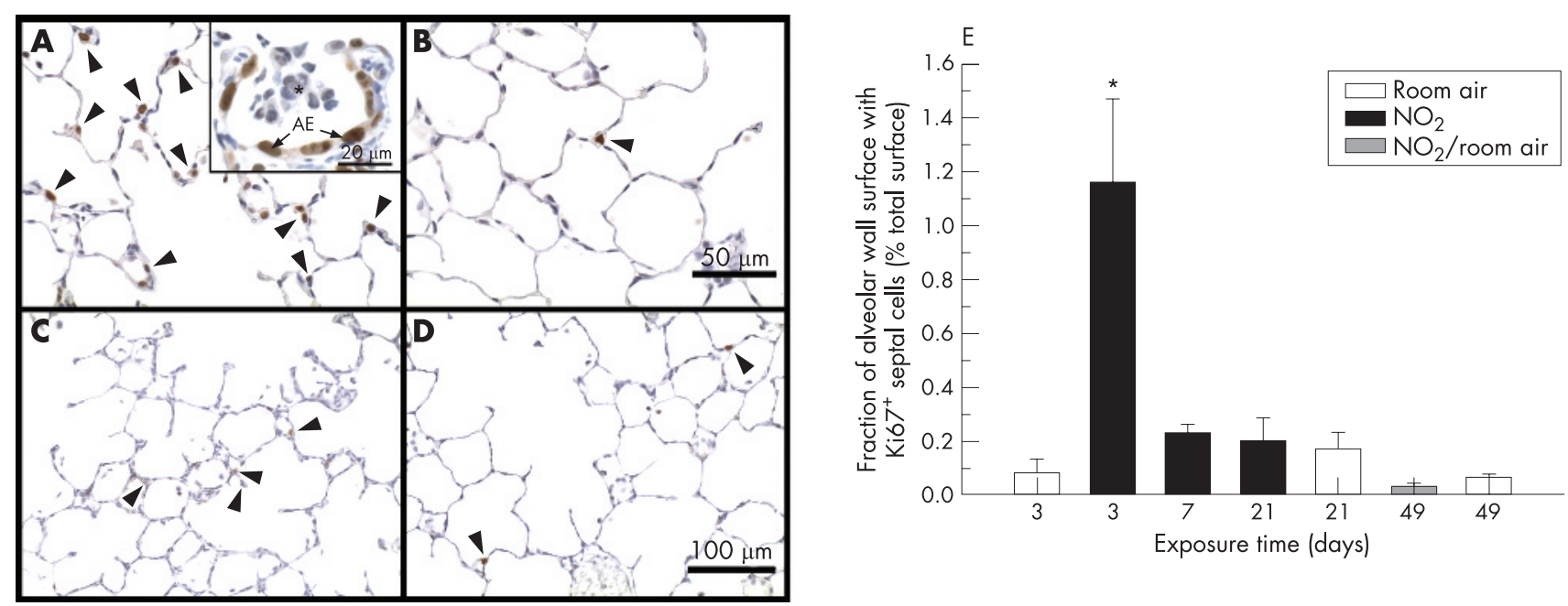

Figure 2 Proliferation of alveolar septal cells. Proliferating alveolar septal wall cells (arrowheads) are shown by indirect immunohistochemistry against proliferation marker Ki-67 in lungs exposed to (A) nitrogen dioxide for 3 days, (B) room air for 3 days, (C) nitrogen dioxide for 7 days and (D) nitrogen dioxide for 21 days. (E) Quantification of Ki-67 staining (as shown in A-D) shows a significant increase in proliferation of structure-forming alveolar septal wall cells at day 3 of exposure to nitrogen dioxide. Insert in (A) shows that most Ki-67 positive cells are alveolar epithelial cells $(A, E)$; asterisk denotes inflammatory cells in the alveolar airspace. The magnifications used in $(A)$ and $(B)$, and in $(C)$ and $(D)$, were the same. Significant differences between groups ( $p<0.05$, one-way analysis of variance followed by post hoc multiple comparisons according to Tukey's test) are indicated as follows: * vs all groups. 

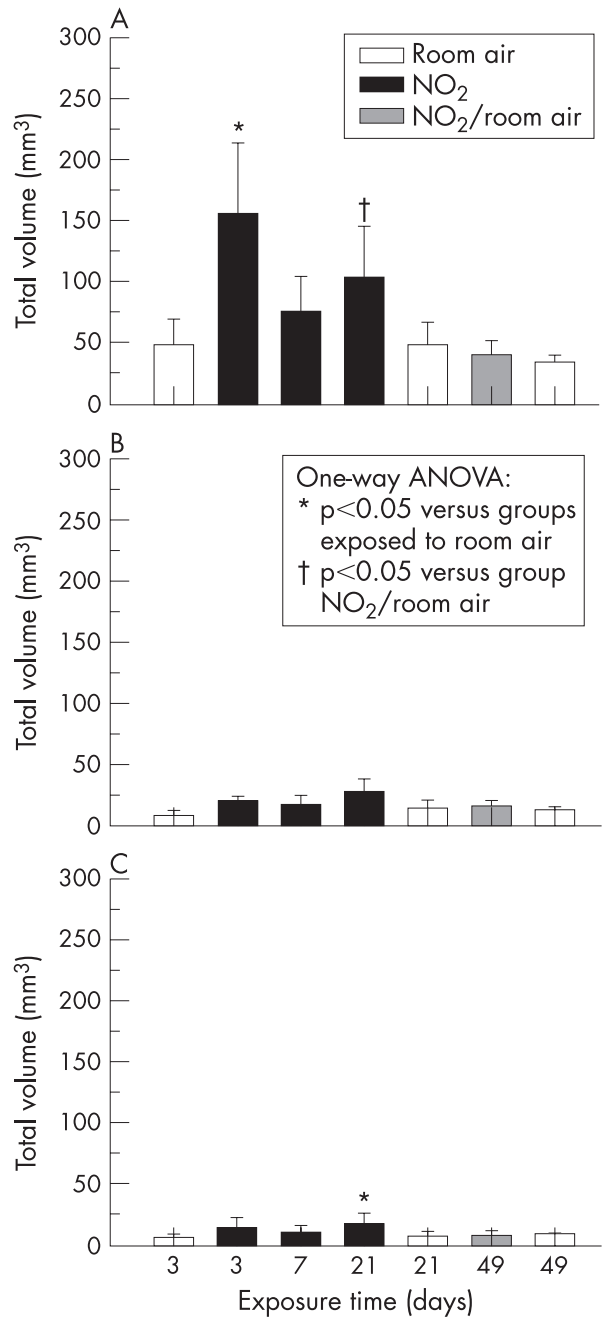

Figure 3 Quantification of total volumes of inflammatory cells (performed on $1 \mu \mathrm{m}$ thick glycol methacrylate sections) shows (A) an increase in alveolar macrophages in lungs exposed to nitrogen dioxide for 3 and 21 days, (B) a slight but insignificant increase in polymorphonuclear granulocytes after 21 days of exposure to nitrogen dioxide, and (C) a significant increase in other inflammatory cells at day 21 of exposure to nitrogen dioxide. Significant differences between groups ( $p<0.05$, oneway analysis of variance followed by post hoc multiple comparisons according to Tukey's test) are indicated as follows: * vs all groups exposed to room air and vs $\mathrm{NO}_{2}$ /room air; $†$ vs $\mathrm{NO}_{2} /$ room air.

During the following 2 weeks, they had a weight gain of about $20 \mathrm{~g}$, whereas control rats exposed to room air for the same period of time had an increase in body weight of about $70 \mathrm{~g}$. This was because of a significantly lower amount of mean food consumption in rats exposed to nitrogen dioxide, which was $36 \%$ of the mean food consumption of control animals during the first 3 days of exposure and about $70 \%$ during the last 2 weeks (table 1). Animals kept in room air for 28 days after 21 days of exposure to nitrogen dioxide consumed considerably more food than during exposure to nitrogen dioxide, and achieved a body weight of 280 (11) g at the end of the experiment, which was not significantly different from the mean body weight of 297 (13) g of the control rats exposed to room air for 49 days (table 1 ).

\section{Apoptosis and proliferation of alveolar septal cells}

In rat lungs exposed to nitrogen dioxide for 3 days, numerous apoptotic cells were observed in alveolar septal walls and in the alveolar space by TUNEL assay (fig lA) and immunohistochemistry for active caspase 3 (fig 1B). By contrast, apoptotic cells were extremely rare in lungs of animals exposed to room air (not shown). At day 3 of exposure to nitrogen dioxide, the fraction of alveolar surface area that was associated with apoptotic cells of the alveolar septal wall was significantly increased to more than eightfold in comparison with agematched control animals (fig lE). This increase exclusively reflects the increase in apoptosis of alveolar septal wall cells, as free apoptotic cells present in the alveolar air space were not considered in our analysis. With increasing exposure time, a continuous decrease of apoptotic events in alveolar septal wall cells was recorded. However, the level of alveolar septal wall apoptosis remained significantly higher than in control animals exposed to room air.

Double stainings using the epithelial and microvascular endothelial markers surfactant protein $\mathrm{D}$ and aquaporin $\mathrm{l}$ showed that most of the apoptotic cells were of epithelial origin (fig lC). Apoptosis of microvascular endothelial cells was not observed (fig 1D).

Immunohistochemistry for the proliferation marker Ki-67 (fig 2A-D) showed large numbers of proliferating cells at day 3 of exposure to nitrogen dioxide. The fraction of alveolar surface area associated with proliferating alveolar septal cells was significantly increased to approximately 14 -fold above the level of the respective age-matched control group (fig 2E). At day 7 , the number of proliferating cells had dramatically decreased and was no longer significantly different from control group levels.

Correlation analysis (performed in nitrogen dioxide-exposed lungs only) showed a significant positive correlation $(\mathrm{r}=0.604$, $\mathrm{p}=0.008$ ) between apoptosis and proliferation of structure forming alveolar septal wall cells, whereas no relationship was seen with any parameter in the inflammatory cells.

\section{Inflammation}

Rat lungs exposed to nitrogen dioxide for 3 days had a threefold increase in the total volume of alveolar macrophages compared with lungs exposed to room air (fig 3A), whereas polymorphonuclear granulocytes were only slightly but insignificantly increased (fig 3B). After 21 days of exposure to nitrogen dioxide, the total volumes of alveolar macrophages and of other inflammatory cells were increased by about twofold (fig 3A,C).

\section{Airspace enlargement}

Control lungs exposed to room air showed a progressive increase in airspace size with age (fig 4A-E). In comparison with age-matched control lungs, animals exposed to nitrogen dioxide for 3 days showed normal indices of airspace size - that is, mean airspace chord length, alveolar surface density and volume-weighted mean airspace volume, respectively (table 2 ). However, airspace enlargement was evident at day 7 of exposure to nitrogen dioxide as indicated by significant increases in mean airspace chord length, surface density and volume-weighted mean airspace volume. Airspace enlargement further progressed until day 21: lungs exposed to nitrogen dioxide had an increase in mean airspace chord length of about $20 \%$ and in volume-weighted mean airspace volume of about $40 \%$ in comparison with age-matched control lungs. Although the mean chord length was considerably decreased in lungs exposed to nitrogen dioxide for 21 days followed by 28 days exposure to room air in comparison with lungs exposed to nitrogen dioxide for 21 days, volume-weighted mean airspace volume and surface density were not markedly different between these two groups (table 2). Hence, airspace enlargement appeared to persist after termination of exposure to 

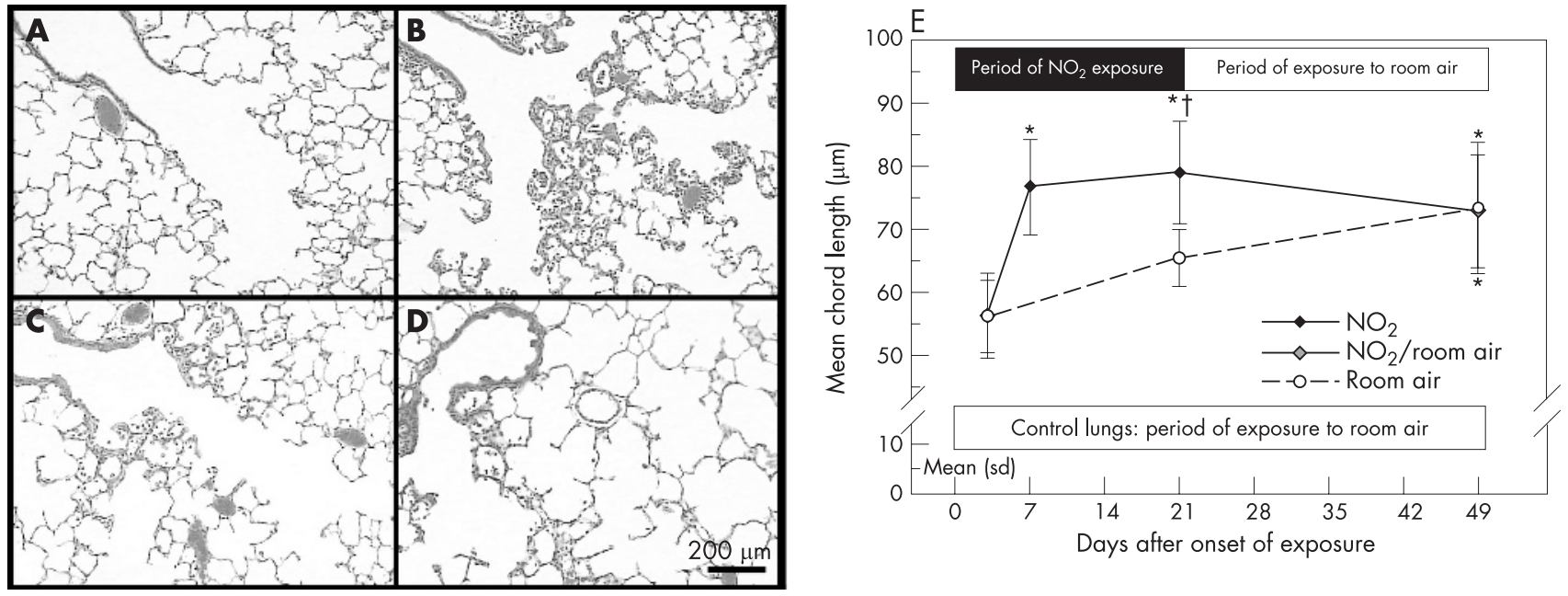

Figure 4 Histopathology of terminal bronchioles and gas exchange area. H\&E stainings of paraffin sections show (A) normal histology in lungs exposed to room air for 21 days, whereas lungs exposed to nitrogen dioxide for (B) 3 days, (C) 7 days and (D) 21 days show inflammation and airspace enlargement. (E) Quantification of mean chord length as an indicator of changes in airspace size (performed on 1 um thick glycol methacrylate sections) shows a continuous increase in mean chord length in control lungs and an accelerated increase in lungs exposed to nitrogen dioxide. All micrographs were taken at identical magnification. Significant differences between groups $(p<0.05$, one-way analysis of variance followed by post hoc multiple comparisons according to Tukey's test) are indicated as follows: * vs room air 3 days and $\mathrm{NO}_{2} 3$ days; tvs room air 21 days.

nitrogen dioxide. Equivalent indices of airspace size were noted for age-matched animals exposed to room air for 49 days. The major difference between lungs exposed to nitrogen dioxide versus room air was that the airspace size of lungs exposed to nitrogen dioxide had already reached values that were equivalent to 49 days control lungs by day 7 .

\section{Loss of alveolar walls}

Despite the fact that airspace enlargement was prominent in lungs exposed to nitrogen dioxide for 21 days compared with age-matched control lungs, we did not find any sign of a loss of alveolar walls. By contrast, absolute volume and total surface area of alveolar septal walls were increased in nitrogen dioxideexposed rats (day 21 ) by $53 \%$ and $29 \%$, respectively, compared with age-matched controls (table 2, figs 5 and 6). Alveolar septal wall volume continued to increase after proliferation and apoptosis had returned to normal. There were no significant differences in the absolute volume of the non-parenchymal compartment. The increases in volume and surface area of alveolar septal walls were even more prominent when massspecific parameters are considered (data not shown). Quantitative ultrastructural analysis showed that the increased alveolar septal wall volume at day 3 of nitrogen dioxide exposure was exclusively due to an increase in the volume of

Table 2 Quantitative morphological data obtained by light microscopy: absolute volumes $\left(\mathrm{mm}^{3}\right)$

\begin{tabular}{|c|c|c|c|c|c|c|c|}
\hline \multirow[b]{2}{*}{ Absolute volumes $\left(\mathrm{mm}^{3}\right)$} & \multicolumn{3}{|l|}{ Room air } & \multicolumn{3}{|l|}{$10 \mathrm{ppm} \mathrm{NO}$} & \multirow{2}{*}{$\frac{\mathrm{NO}_{2} / \text { room air }}{21 \text { and } 28 \text { days }}$} \\
\hline & 3 days & 21 days & 49 days & 3 days & 7 days & 21 days & \\
\hline Fixed lung & 4920 (549) & $5496(435)$ & $7140(213)^{*}$ & $4768(592)$ & $6322(661) \dagger$ & $7623(514) \ddagger$ & 7665 (1107)‡ \\
\hline Non-parenchyma & $689(76)$ & $703(104)$ & $725(86)$ & $626(108)$ & $758(177)$ & $733(62)$ & 752 (129) \\
\hline Parenchyma & $4231(547)$ & $4793(352)$ & $6415(190)^{*}$ & $4142(564)$ & $5564(553) \dagger$ & $6891(501) \ddagger$ & $6913(1002) \ddagger$ \\
\hline Airspace & $3768(525)$ & $4316(332)$ & $5765(204)$ & $3546(556)$ & $4941(486)$ & $6161(487)$ & $6275(962) \uparrow$ \\
\hline Alveolar macrophages & $47(22)$ & $47(20)$ & $39(12)$ & $155(59)^{* *}$ & $75(29)$ & $103(43) \S$ & $34(6)$ \\
\hline Granulocytes (PMNs) & $5.6(2.8)$ & $7.1(3.2)$ & $7.4(3.3)$ & $13.6(7.8)$ & $9.9(5.5)$ & $16(8.9)$ & $7.8(0.3)$ \\
\hline Other cells & $8.2(3.6)$ & $14(6.8)$ & $15.4(5.1)$ & $19.2(4.4)$ & $16.8(7.4)$ & $27.3(11)^{* *}$ & $13(2.3)$ \\
\hline Alveolar septal wall & $463(62)$ & $477(40)$ & $650(52)$ & $596(86)$ & 624 (112) & $729(93)^{\top}$ & $638(74)^{\oplus}$ \\
\hline Mean thickness $(\mu \mathrm{m})$ & $4.2(0.5)$ & $4(0.2)$ & $4.1(0.4)$ & $5.3(0.6)^{* *}$ & $5(0.5)^{* *}$ & $4.7(0.4)$ & $4.2(0.3)$ \\
\hline Mean chord length $(\mu \mathrm{m})$ & $56.1(5.7)$ & $65.5(4.4)$ & $73.3(10.2) t \dagger$ & $56.2(6.7)$ & $76.6(7.6) \mathrm{tt}$ & $78.9(8)^{*}$ & $72.8(9)+t$ \\
\hline $\begin{array}{l}\text { Volume-weighted mean airspace } \\
\text { volume }\left(10^{6} \mu \mathrm{m}^{3}\right)\end{array}$ & $4.83(1.35)$ & $5.36(1.92)$ & $6.36(1.92)$ & $3.17(0.45)$ & $6.88(2.11)^{\bullet}$ & $7.49(0.88)$ & $7.15(0.73) \oplus$ \\
\hline Surface density $(1 / \mathrm{mm})$ & $52.4(3.7)$ & $49.7(5.3)$ & $48.7(2.7)$ & $54.9(6.1) \S \S$ & 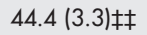 & $44.8(4.1) \ddagger \ddagger$ & $45.3(2.3) \ddagger \ddagger$ \\
\hline Total alveolar surface area $\left(\mathrm{cm}^{2}\right)$ & $2205(214)$ & $2377(240)$ & $3125(161) \pm$ & $2253(180)$ & $2479(384)$ & $3078(269) \ddagger$ & $3121(433) \pm$ \\
\hline
\end{tabular}

Significant differences among groups at $\mathrm{p}<0.05$ (one-way analysis of variance followed by post hoc pairwise multiple comparisons, Tukey's test) are indicated as follows:

*vs room air 3 days, 21 days; $\mathrm{NO}_{2} 3$ days.

tvs room air 3 days, 21 days; $\mathrm{NO}_{2} 3$ days, 21 days; $\mathrm{NO}_{2} /$ room air.

†vs room air 3 days, 21 days; $\mathrm{NO}_{2} 3$ days, 7 days.

§vs $\mathrm{NO}_{2}$ /room air.

Tvs room air 3 days, 21 days.

**vs room air 3 days, 21 days, 49 days; $\mathrm{NO}_{2} /$ room air.

††vs room air 3 days; $\mathrm{NO}_{2} 3$ days.

\#¥vs $\mathrm{NO}_{2} 3$ days.

$\S \S v s \mathrm{NO}_{2} 7$ days, 21 days; $\mathrm{NO}_{2}$ /room air. 


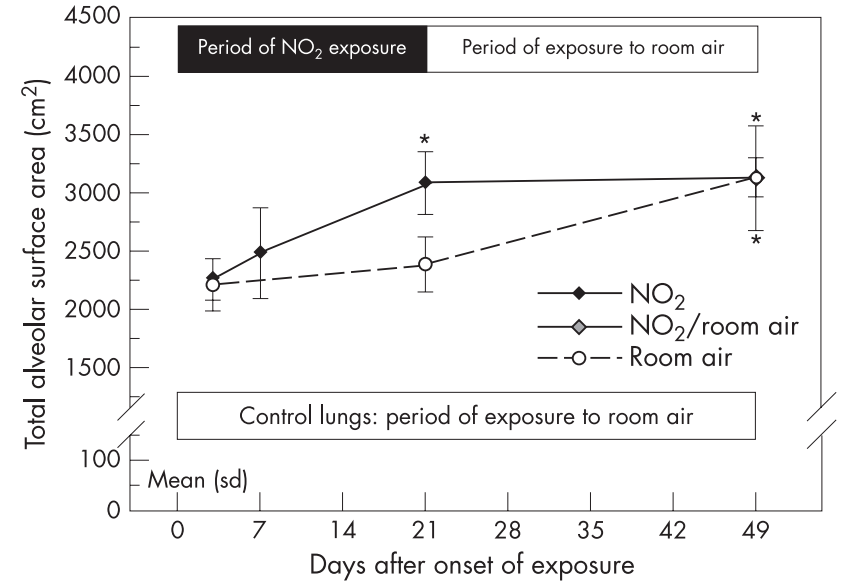

Figure 5 Quantification of total alveolar surface area as an indicator of loss of alveolar walls (performed on $1 \mu \mathrm{m}$ thick glycol methacrylate sections) shows a continuous increase in total alveolar wall surface in control lungs and an accelerated increase in lungs exposed to nitrogen dioxide. Significant differences between groups ( $p<0.05$, one-way analysis of variance followed by post hoc multiple comparisons according to Tukey's test) are indicated as follows: *vs room air 3 days, 21 days and vs $\mathrm{NO}_{2} 3$ days, 7 days.

the alveolar epithelium (fig 7). Whereas no further increase was noted in the alveolar epithelial volume of nitrogen dioxideexposed lungs, the volumes of capillary endothelium and interstitial tissue as well as of collagen and elastin were increased at day 21 compared with age-matched control lungs. Notably, the collagen-to-elastin ratio was significantly lower in nitrogen dioxide-exposed lungs at day 21 compared with control lungs exposed to room air for 21 days (fig 8).

A progressive age-dependent increase was noted in control lungs in all structural parameters related to the gas exchange region. Notably, total alveolar surface area and absolute alveolar septal volume of nitrogen dioxide-exposed rats (day 21) were largely equivalent to values characteristic of control animals at day 49. There was no noticeable change in alveolar septal wall volume and total alveolar surface area after exposure to nitrogen dioxide had been terminated, and no changes were noted after an additional exposure to room air for 28 days.

\section{DISCUSSION}

The effects of exposure of experimental animals to nitrogen dioxide vary widely-for example, with the species and strain exposed, the concentration and duration applied, as well as age and sex of the animals-as comprehensively reviewed by others. ${ }^{25}$ In our study of juvenile male Fischer 344 rats, which were $8-10$ weeks of age at the beginning of the experiments, lungs exposed to an atmosphere containing $10 \mathrm{ppm}$ nitrogen dioxide for $23 \mathrm{~h}$ per day for 3 days showed an increased turnover of alveolar septal cells indicated by an eightfold increase in apoptotic events and a 14 -fold increase in proliferation in comparison with age-matched control lungs. Lungs exposed to nitrogen dioxide for 21 days exhibited quantitative structural characteristics equivalent to control lungs, which were 28 days older. As transfer of lungs to room air after 21 days of exposure to nitrogen dioxide did not result in a reversal of this process, we suggest that nitrogen dioxide induced an accelerated growth of the lung, which, however, was associated with an imbalance in the relative composition of the extracellular matrix indicated by a decreased collagen-toelastin ratio.

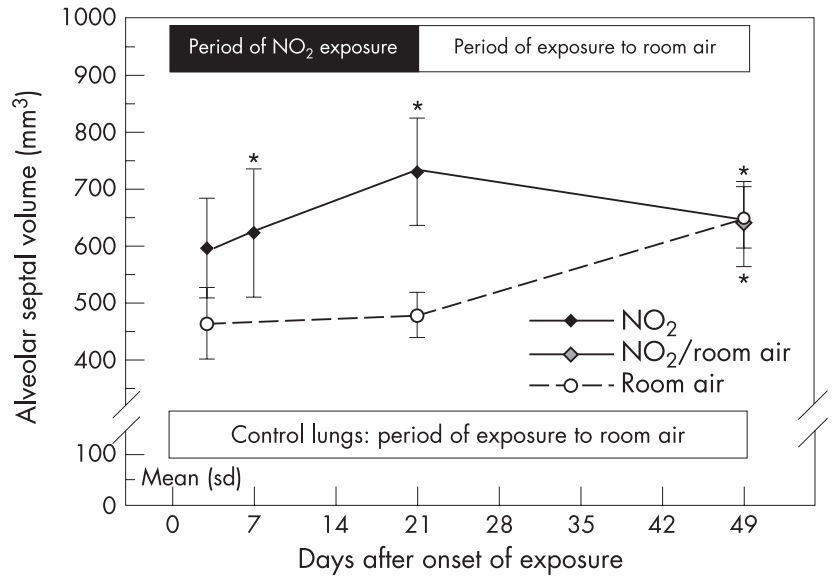

Figure 6 Quantification of total alveolar septal volume as an indicator of a potential loss of alveolar walls (performed on $1 \mu \mathrm{m}$ thick glycol methacrylate sections) shows an increase in alveolar wall volume in control lungs and an accelerated increase in lungs exposed to nitrogen dioxide. Significant differences between groups $(p<0.05$, one-way analysis of variance followed by post hoc multiple comparisons according to Tukey's test) are indicated as follows: *vs room air 3 days, 21 days.

In agreement with results from in vitro studies, ${ }^{17}$ we observed a significant increase in alveolar septal wall cell death in lungs exposed to nitrogen dioxide in vivo. Double stainings for apoptosis and markers of alveolar cell types showed that alveolar epithelial type II cells were the main target of nitrogen dioxide-induced apoptotic cell death, whereas vascular and capillary endothelial cells were not affected. Although experimental induction of epithelial and/or endothelial cell apoptosis has been linked to the development of airspace enlargement in various murine models ${ }^{5-7}$ including cigarette smoke exposure, ${ }^{8}$ apoptosis of alveolar epithelial cells appeared to predominate in human emphysema. ${ }^{11}{ }^{11}$ Recent studies have shown that in the lungs of patients with emphysema, however, proliferation of alveolar septal cell was also increased. ${ }^{10}$ These findings indicate that emphysema may not simply be the consequence of the induction of apoptosis, but is more likely to be the result of an imbalance between apoptosis and proliferation in humans. ${ }^{9} 10$

Exposure of rats to nitrogen dioxide was shown to induce airway inflammation which, as in human COPD, was dominated by alveolar macrophages and neutrophilic granulocytes. ${ }^{15} 16$ Only a minor contribution of polymorphonuclear granulocytes was seen in our experiments. Whereas some studies have reported that exposure to nitrogen dioxide may result in emphysema, ${ }^{16}$ others have not found evidence for this. ${ }^{26}$ Unfortunately, all these studies relied only on the measurement of airspace enlargement to assess the presence of emphysema. An increase in airspace size alone, however, is insufficient to conclude that emphysema has developed. ${ }^{27}$ Stereological approaches to quantify emphysema has shown that, in human emphysema ${ }^{28}$ as well as in elastase-induced emphysema in the mouse, ${ }^{29}$ airspace enlargement is accompanied by a loss of alveolar walls as evidenced by a decrease in total alveolar surface area and total alveolar wall volume. Using a stereological approach to quantify airspace enlargement by independent measurements of mean airspace chord length, alveolar surface density ( $\mathrm{S} / \mathrm{V}$ ratio), and volume-weighted mean airspace volume, respectively, as well as quantification of total alveolar surface area and absolute alveolar septal wall volume, we showed that increased airspace enlargement during exposure to nitrogen dioxide was not associated with a loss of alveolar walls. On the contrary, lungs exposed to nitrogen 

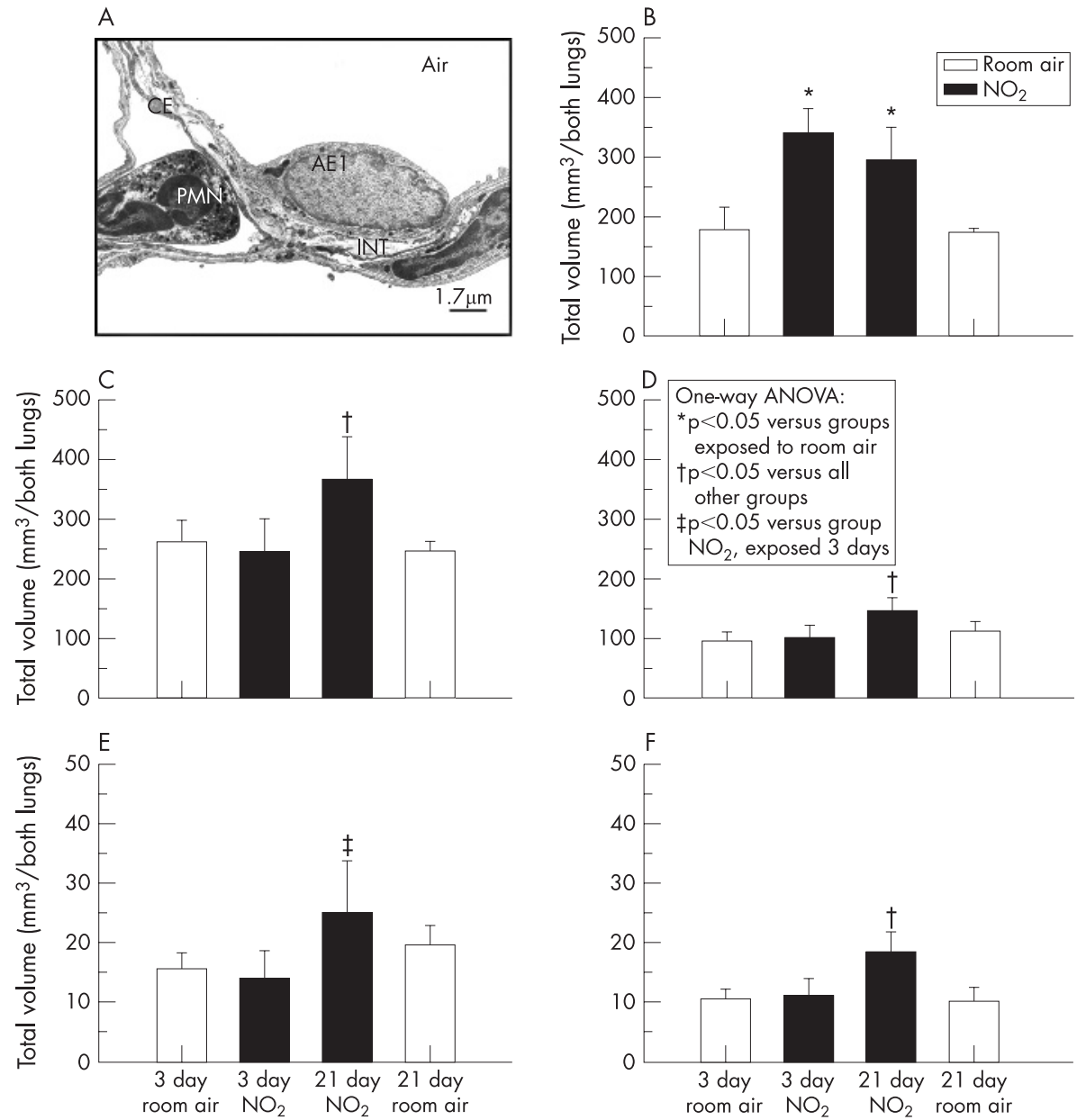

Experimental groups

Experimental groups

Figure 7 (A) Ultrastructure of alveolar septal wall of lung exposed to room air for 3 days. Electron microscopic quantification of different components of the alveolar septal wall showed an increase in the total volume of (B) the alveolar epithelium already on day 3 of exposure to nitrogen dioxide whereas the other components of the alveolar septal wall were not affected. At day 21 of exposure to nitrogen dioxide, an equivalent increase in volume was seen in all components of the alveolar wall; (B) alveolar epithelium, (C) interstitial tissue, (D) capillary endothelium, (E) collagen fibres and (F) elastin. Significant differences between groups ( $p<0.05$, one-way analysis of variance followed by post hoc multiple comparisons according to Tukey's test) are indicated.

dioxide for 21 days were characterised by a $29 \%$ increase in total alveolar surface area and a 53\% increase in absolute septal volume in comparison with age-matched control lungs.
Hence, relying on indicators of airspace enlargement alone would have led to the false conclusion that nitrogen dioxide induced apoptosis with subsequent emphysema. By contrast,
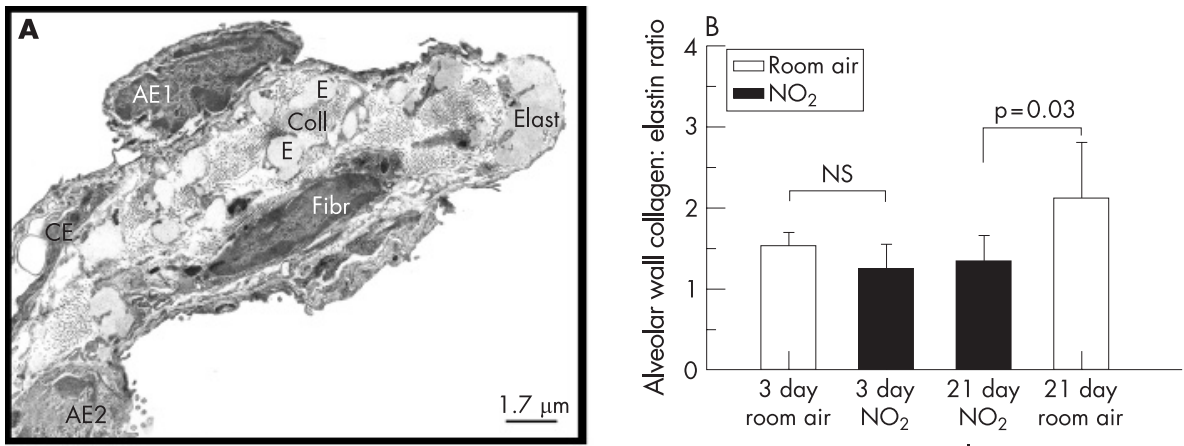

Experimental groups

Figure 8 (A) Ultrastructure of extracellular matrix composition of alveolar septal wall entrance ring of lung exposed to room air for 3 days. (B) Electron microscopic quantification showed a significantly decreased collagen-to-elastin ratio in lungs exposed to nitrogen dioxide for 21 days compared with control lungs exposed to room air for 21 days. At day 3, no differences were seen between lungs exposed to nitrogen dioxide or room air. Significant differences between groups ( $<<0.05$, Student's $t$ test) are indicated. $A E 1$, alveolar epithelial type 1 cell; $A E 2$, alveolar epithelial type 2 cell; $C E$, capillary endothelium; Coll, collagen fibrils; E, Elast, elastin fibres; Fibr, fibrocytes. 
inclusion of indicators of loss of alveolar walls-for example, total alveolar wall volume and total alveolar surface areashowed that the increase in apoptotic events observed in lungs exposed to nitrogen dioxide was over-compensated by the parallel increase in proliferation.

In early studies, Evans and coworkers showed that nitrogen dioxide results in hyperplasia of alveolar epithelial type II cells as a response to the initial damage of type I cells. ${ }^{30}$ In line with this, our quantitative ultrastructural analysis showed that, at day 3 of exposure to nitrogen dioxide only the alveolar epithelium exhibited an increase in the total volume, while all other compartments of the alveolar septal wall were unaffected. Hyperplasia of alveolar epithelial type II was followed by transformation of type II into type I cells. ${ }^{18}$ Although transformation is one mechanism for resolving hyperplasia, apoptosis of type II cells, as shown in this study, is another mechanism which is probably more important in quantitative terms than transformation. ${ }^{31}$ The significant correlation between the indices of proliferating and apoptotic cells in our study highlights the close link between apoptosis and proliferation of alveolar septal wall cells in this model. The decrease in both proliferation and apoptosis with exposure time may reflect some adaptive mechanisms induced-for example, in alveolar epithelial type II cells, which were previously shown to exhibit restored antioxidant superoxide dismutase acitivity at day $20,{ }^{32}$ and/or in bronchoalveolar lavage cells, which showed an increase in glutathione peroxidase and superoxide dismutase enzyme activities in this model. ${ }^{33}$ However, we cannot discriminate whether the induction of these protective mechanisms were direct effects due to exposure or an indirect effect due to the reduced food intake (see below).

After 21 days of exposure to nitrogen dioxide, all compartments of the alveolar septum exhibited an increase in their total volume in comparison with room air exposure. However, no equivalent increase was seen in the collagen-to-elastin ratio, indicating that accelerated lung growth was associated with an imbalance in extracellular matrix deposition.

In this study, as in most studies performed in the field of emphysema research, ${ }^{6-8}$ animals were $8-10$ weeks of age at the beginning of exposure-that is, they were juveniles. Considerable normal lung growth is observed in rats and mice up to an age of at least 5 months, and comprises an increase in total alveolar surface area and absolute alveolar wall volume as well as an age-dependent increase in airspace size. ${ }^{34} 35$ The quantitative morphological data obtained in our study of control animals compare well with data published on ageing male Fischer 344 rats summarised recently. ${ }^{35}$ In this review, the authors pointed out that only small changes occur in tissue volumes from 5 to 26 months of age with the exception of a reduction in the extracellular matrix component of the interstitium, whereas total airspace volume and airspace size increased with age. ${ }^{35}$ These structural changes are accompanied by age-related changes in the biochemical composition of the extracellular matrix as well as in lung function. ${ }^{36}$ Age-related changes in airspace size are also well documented in humans, ${ }^{37}$ as are age-related changes in lung function. ${ }^{38}$ Both an agedependent decline in lung function and an increase in airspace size are significantly accelerated in smokers. ${ }^{39}{ }^{40}$ Notably, data from a recent prospective study of California schoolchildren suggest that ambient nitrogen dioxide is a major contributor to the adverse effects of air pollution on lung development from the age of 10 to 18 years, which lead to clinically significant deficits-for example, in forced expiratory volume in $1 \mathrm{~s}$ as children reached adulthood ${ }^{41}$ Although in the present study lung function parameters were not analysed, we have recently shown that exposure to nitrogen dioxide resulted in a progressive decline in expiratory airflow associated with an increase in airspace size as well as in volume-weighted mean alveolar volume in juvenile mice. ${ }^{42}$

From our study we cannot discriminate if the changes observed were a direct effect of the exposure to nitrogen dioxide or an indirect effect due to the significantly reduced food consumption (day 3: $36 \%$, day 7: $44 \%$ of control animals at day 3 ; day 21 : $69 \%$ of control animals at day 21 ). Calorie restriction (two thirds reduction for 2 weeks) in mice has recently been shown to result in a reduction in body weight of about $40 \%$ with a concomitant increase in apoptosis, a $25 \%$ reduction in total alveolar surface area and a reduction in total alveolar wall volume of about one third. ${ }^{43}$ Unlike these findings, exposure of rat lungs to nitrogen dioxide did not result in loss of body weight but only in a reduction in weight gain which may be due to the reduced activity, and hence metabolism, of nitrogen dioxide-exposed animals. We did not observe a loss of alveolar walls as a direct effect of exposure or as an indirect effect due to reduced food intake. Instead, a 29\% increase in total alveolar surface area and a 53\% increase in total alveolar wall volume were observed after 21 days of exposure to nitrogen dioxide versus room air. Wright and Churg ${ }^{44}$ pointed out that all of the animal models of emphysema using cigarette smoke exposure that have evaluated changes in body weight have shown a failure of smoke-exposed animals to gain weight. In a recent pair-fed study in mice, cigarette smoking for 4 weeks was shown to result in reduced weight gain equivalent to a food restriction group. In contrast with food restriction, smoke exposure caused a reduction in hypothalamic neuropeptide $\mathrm{Y}$ and fat mass, and regulated adipose cytokines, finally resulting in the reduced weight gain. ${ }^{45}$ Unfortunately, data regarding inflammation or histopathology were not recorded in this study. From human studies it is well documented that, in contrast with chronic starvation well-nourished adults who, for experimental or other reasons, have lowered their food intakes an apparently enhanced metabolic efficiency is observed resulting from changes in metabolic rates, which are disproportionate to the changes in body weight. ${ }^{46}$

In conclusion, nitrogen dioxide exposure of juvenile rats resulted in increased alveolar septal cell turnover as evidenced by an increase in apoptosis and proliferation of alveolar septal cells. Comparison with age-matched control lungs indicates that increased cell turnover was associated with an acceleration of normal lung growth as evidenced by an increase in airspace size, total alveolar surface area as well as total alveolar wall volume. Despite the increase in both collagen and elastin, the reduced collagen-to-elastin ratio in nitrogen dioxide-exposed animals indicates that extracellular matrix deposition was impaired and may result in altered lung mechanics in this model. We suggest that the changes observed are compatible with an accelerated lung growth of rat lungs exposed to nitrogen dioxide which, however, does not comprise a proportional growth of collagen and elastin. Exposure of juvenile rats to nitrogen dioxide may be a useful model to study mechanisms leading to the adverse effects of air pollution on postnatal lung development observed in children and young adults. ${ }^{41}$

\section{ACKNOWLEDGEMENTS}

We acknowledge the help of Bernd Müller and Petra Staats with the nitrogen dioxide exposure model, and the expert technical assistance of Roswitha Naumann and Tanja Rausch in all steps of organ preparation, tissue processing, sectioning and staining procedures.
Authors' affiliations
Heinz Fehrenbach, Gregor Zimmermann, Ellen Starke, Vlad A Bratu, Dominik Conrad, Ali Ö Yildirim, Antonia Fehrenbach, Clinical Research Group "Chronic Airway Diseases", Department of Internal Medicine (Respiratory Medicine), Philipps-University, Baldingerstrasse, D-35043 Marburg, Germany 
Funding: The study was supported by a grant from the German Ministerium für Bildung und Forschung (FKZ 01GC0103).

Competing interests: None.

\section{REFERENCES}

1 American Thoracic Society. Standards for the diagnosis and care of patients with chronic obstructive pulmonary disease. Am J Respir Crit Care Med 1995; 152:S77-121

2 Hogg JC, Senior RM. Chronic obstructive pulmonary disease-part 2: pathology and biochemistry of emphysema. Thorax 2002;57:830-4.

3 Shapiro SD. Vascular atrophy and VEGFR-2 signaling: old theories of pulmonary emphysema meet new data. J Clin Invest 2000;106:1309-10.

4 Tuder RM, Petrache I, Elias JA, et al. Apoptosis and emphysema: the missing link. Am J Respir Cell Mol Biol 2003;28:551-4.

5 Kasahara Y, Tuder RM, Taraseviciene-Stewart L, et al. Inhibition of VEGF receptors causes lung cell apoptosis and emphysema. J Clin Invest 2000;106:1311-19.

6 Tang K, Rossiter HB, Wagner PD, et al. Lung-targeted VEGF inactivation leads to an emphysema phenotype in mice. J Appl Physiol 2004;97:1559-66.

7 Aoshiba K, Yokohori N, Nagai A. Alveolar wall apoptosis causes lung destruction and emphysematous changes. Am J Respir Cell Mol Biol 2003;28:555-62.

8 Ma B, Kang MJ, Lee CG, et al. Role of CCR5 in IFN-gamma-induced and cigarette smoke-induced emphysema. J Clin Invest 2005;1 15:3460-72.

9 Imai K, Mercer BA, Schulman LL, et al. Correlation of lung surface area to apoptosis and proliferation in human emphysema. Eur Respir J 2005;25:250-8.

10 Yokohori N, Aoshiba K, Nagai A. Increased levels of cell death and proliferation in alveolar wall cells in patients with pulmonary emphysema. Chest 2004; 125:626-32.

11 Kasahara Y, Tuder RM, Cool CD, et al. Endothelial cell death and decreased expression of vascular endothelial growth factor and vascular endothelial growth factor receptor 2 in emphysema. Am J Respir Crit Care Med 2001;163:737-44.

12 Barnes PJ. Mediators of chronic obstructive pulmonary disease. Pharmacol Rev 2004;56:515-48.

13 Foronjy RF, Mercer BA, Maxfield MW, et al. Structural emphysema does not correlate with lung compliance: lessons from the mouse smoking model. Exp Lung Res 2005;31:547-62.

14 Gas Research Institute. Characterization of emission rates from indoor combustion sources. Chicago, Illinois: 1985, GRI 85/, 0075

15 Garn H, Siese A, Stumpf S, et al. Shift toward an alternatively activated macrophage response in lungs of NO2-exposed rats. Am J Respir Cell Mol Biol 2003;28:386-96.

16 Glasgow JE, Pietra GG, Abrams WR, et al. Neutrophil recruitment and degranulation during induction of emphysema in the rat by nitrogen dioxide. Am Rev Respir Dis 1987; 135:1129-36.

17 Persinger RL, Blay WM, Heintz NH, et al. Nitrogen dioxide induces death in lung epithelial cells in a density-dependent manner. Am J Respir Cell Mol Biol 2001;24:583-90.

18 Evans MJ, Cabral U, Stephens RJ, et al. Transformation of alveolar type 2 cells to type 1 cells following exposure to $\mathrm{NO}_{2}$. Exp Mol Pathol 1975;22:142-50.

19 Müller B, Garn H, Hochscheid R. Impaired recycling of surfactant-like liposomes in type II pneumocytes from injured lungs. Thorax 2003;58:127-34.

20 Fehrenbach $\mathrm{H}$, Ochs M. Studying lung ultrastructure. In: Uhlig S, Taylor AE, eds. Methods in pulmonary research. Basel: Birkhäuser Verlag, 1998:429-54.

21 Fehrenbach $\mathbf{H}$, Fehrenbach A, Pan T, et al. Keratinocyte growth factor-induced proliferation of rat airway epithelium is restricted to Clara cells in vivo. Eur Respir J 2002;20:1185-97.
22 Fehrenbach H, Kasper M, Koslowski R, et al. Alveolar epithelial type II cell apoptosis in vivo during resolution of keratinocyte growth factor-induced hyperplasia in the rat. Histochem Cell Biol 2000;1 14:49-61.

23 Kageyama $M$, Takagi $M$, Parmley RT, et al. Ultrastructural visualization of elastic fibres with a tannate-metal salt method. Histochem J 1985;17:93-103.

24 Fehrenbach $\mathrm{H}$, Schepelmann D, Albes JM, et al. Pulmonary ischemia/ reperfusion injury: a quantitative study of structure and function in isolated heartlungs of the rat. Anat Rec 1999;255:84-9.

25 Persinger RL, Poynter ME, Ckless K, et al. Molecular mechanisms of nitrogen dioxide induced epithelial injury in the lung. Mol Cell Biochem 2002;2345:71-80.

26 Mercer RR, Costa DL, Crapo JD. Effects of prolonged exposure to low doses of nitric oxide or nitrogen dioxide on the alveolar septa of the adult rat lung. Lab Invest 1995:73:20-8.

27 Fehrenbach $\mathrm{H}$. Animal models of chronic obstructive pulmonary disease: some critical remarks. Pathobiology 2002;70:277-83

28 Wiebe BM, Laursen $\mathrm{H}$. Lung morphometry by unbiased methods in emphysema: bronchial and blood vessel volume, alveolar surface area and capillary length. APMIS 1998;106:651-6.

29 Heemskerk-Gerritsen BA, Dijkman JH, ten Have-Opbroek AA. Stereological methods: a new approach in the assessment of pulmonary emphysema. Microsc Res Tech 1996;34:556-62.

30 Evans MJ, Cabral L, Stephens RJ, et al. Renewal of alveolar epithelium in the rat following exposure to $\mathrm{NO}_{2}$. Am J Pathol 1973;70:175-98.

31 Fehrenbach $\mathbf{H}$, Kasper $M$, Tschernig $T$, et al. Keratinocyte growth factor-induced hyperplasia of rat alveolar type II cells in vivo is resolved by differentiation into type I cells and by apoptosis. Eur Respir J 1999; 14:534-44.

32 Hochscheid R, Heinrichs S, Kranz S, et al. Differences in mRNA expression, protein content, and enzyme activity of superoxide dismutases in type II pneumocytes of acute and chronic lung injury. Free Radic Res 2002;36:759-67.

33 Ölker C, Siese A, Stumpf S, et al. Impaired superoxide radical production by bronchoalveolar lavage cells from NO-exposed rats. Free Radic Biol Med 2004;37:977-87.

34 Mizuuchi T, Kida K, Fujino Y. Morphological studies of growth and aging in the lungs of Fischer 344 male rats. Exp Gerontol 1994;29:553-67.

35 Pinkerton KE, Green FHY. Normal aging of the lung. In: Harding R, Pinkerton KE, Plopper C, eds. The lung-development, aging and the environment. London, San Diego: Elsevier Academic Press, 2004:213-33.

36 Sahebjami $\mathbf{H}$. Lung tissue elasticity during the lifespan of Fischer 344 rats. Exp Lung Res 1991;17:887-902.

37 Gillooly M, Lamb D. Airspace size in lungs of lifelong non-smokers: effect of age and sex. Thorax 1993:48:39-43.

38 Pauwels RA, Rabe KF. Burden and clinical features of chronic obstructive pulmonary disease (COPD). Lancet 2004;364:613-20.

39 Scanlon PD, Connett JE, Waller LA, et al. Smoking cessation and lung function in mild-to-moderate chronic obstructive pulmonary disease. The Lung Health Study. Am J Respir Crit Care Med 2000;161:381-90.

40 Hogg JC, Wright JL, Wiggs BR, et al. Lung structure and function in cigarette smokers. Thorax 1994;49:473-8.

41 Gauderman WJ, Avol E, Gilliland F, et al. The effect of air pollution on lung development from 10 to 18 years of age. N Engl J Med 2004;351:1057-67.

42 Wegmann $\mathrm{M}$, Fehrenbach $\mathrm{A}$, Heimann $\mathrm{S}$, et al. $\mathrm{NO}_{2}$-induced airway inflammation is associated with progressive airflow limitation and development of emphysema-like lesions in C57BL/6 mice. Exp Toxicol Pathol 2005;56:341-50

43 De Carlo Massaro G, Radaeva S, Clerch LB, et al. Lung alveoli: endogenous programmed destruction and regeneration. Am J Physiol Lung Cell Mol Physiol 2002;283:305-9.

44 Wright JL, Churg A. Animal models of cigarette smoke-induced COPD. Chest 2002;122:301-6S.

45 Chen $\mathrm{H}$, Hansen MJ, Jones JE, et al. Cigarette smoke exposure reprograms the hypothalamic neuropeptide $\mathrm{Y}$ axis to promote weight loss. Am J Respir Crit Care Med 2006;173:1248-54

46 Shetty PS. Adaptation to low energy intakes: the responses and limits to low intakes in infants, children and adults. Eur J Clin Nutr 1999:53:S14-33.

\section{Let us assist you in teaching the next generation}

Figures from all articles on our website can be downloaded as a PowerPoint slide. This feature is ideal for teaching and saves you valuable time. Just click on the image you need and choose the "PowerPoint Slide for Teaching" option. Save the slide to your hard drive and it is ready to go. This innovative function is an important aid to any clinician, and is completely free to subscribers. (Usual copyright conditions apply.) 